\title{
Molecular profiling for personalized cancer care
}

\author{
Raheela Ashfaq
}

Received: 11 March 2012/ Accepted: 6 May 2012/Published online: 8 June 2012

(C) The Author(s) 2012. This article is published with open access at Springerlink.com

\begin{abstract}
The pace of genomic discoveries in the field of cancer is revolutionizing our understanding of the biological dynamics of cancerous growth and, at the same time, fueling research for newer and smarter cancer therapies to reverse the effects of these alterations. These dynamics are driving a tremendous paradigm shift in cancer diagnostics, drug development and clinical trial design with the hope of eliminating the current structure and approach of cancer care, to one which is driven by the underlying biology of the tumor and, thus highly personalized. Much of this paradigm shift has been fueled by the current availability of novel technologies, platforms and bioinformatic tools. Today, therapies are being rationally designed to target the precise genetic alterations with better clinical outcomes with reduced morbidity. Therefore, molecular profiling of tumors to identify the multiplicity of alterations in a tumor is an essential and necessary companion for targeted therapies.
\end{abstract}

Keywords Molecular profiling - Targets - Targeted therapies $\cdot$ Molecular diagnostics $\cdot$ Clinical trials

\section{Introduction}

Molecular profiling (MP) encompasses the testing of multiple biomarkers to evaluate the underlying genetic alterations present in a tumor at any one point in time [13]. To date molecular profiles may consist of multiple gene mutational analyses, gene copy number changes by

R. Ashfaq $(\bowtie)$

Caris Life Sciences, 6655 North MacArthur Blvd.,

3rd Floor, Irving, TX 75039, USA

e-mail: rashfaq@carisls.com fluorescence in situ hybridization (FISH), gene expression profiles measured by microarrays (MA) and protein expression by immunohistochemistry (IHC). This approach is superior to the testing of one biomarker target which does not take into account the complexity of multiple signaling pathways and cross talk [4].

The cancer literature is replete with studies exploring single biomarkers in clinical trials designed to test single agents or biomarker analyses performed as a post hoc analysis. Although there are a plethora of biomarkers that have emerged that may positively prognosticate or predict response to various therapies, the clinical utility and adoption of this approach has been slow due to validation concerns, reproducibility and translation into clinical care [5-7].

Since comprehensive MP uses a multi-dimensional approach to testing, it is inherently more complex and requires extensive validation. There is significant investment in high cost, high throughput technologies, trained laboratory work force, and laboratory informatics to achieve the level of validation required by CLIA or CAP to offer the test for patient-care. Laboratory developed testvalidations in a CLIA mandated environment typically requires the following [8]:

a. Specimen type and specimen handling protocols: since this variable can hugely affect reproducibility of the test, standardization of specimen handling is imperative. More and more, the formalin fixed paraffin embedded tissue is becoming the sample of choice as it is readily available. This sample type has been shown to perform adequately for mutational analysis; gene expression profiles measured by RT-PCR or oligonucleotide arrays, FISH, and IHC. However, the quality of analysis on this preferred sample type has to be closely monitored based 
on time to fixation, time in fixative and age of the samples. Additionally for newer molecular techniques, internal and external quality checks such as the amount or percentage of tumor nuclei, the quantity and quality of DNA and RNA, measurement of internal housekeeping genes are all important determinants in the overall quality of results [9-15]. Validation of each component of the MP assay, whether being performed in a non-profit hospital-based/academic laboratory or for-profit reference labs, has to follow the strict CLIA guidelines as well as guidelines provided by laboratory associations such as College of American Pathologists (CAP) or CLS1. For each test offered, per validation guidelines, the laboratory must document certain performance characteristics which include:

(a) Accuracy to document that the test produces expected result by appropriate testing of known positive and negative samples. From these accuracy studies the analytical sensitivity, specificity and accuracy of the assay can be determined

(b) Precision studies are performed to determine intra-run and inter-run reproducibility.

(c) The assay will also have to determine appropriate reference ranges and limit of detection for appropriate reporting of results. Ongoing quality assurance and proficiency testing are some other additional requirements by CLIA

Given the resource investment requirements for conducting these multi-dimensional, labor intense assays, it is easily conceivable that these assays are increasingly being offered by large centralized laboratories. (examples include: Genomic Health Inc., Pathwork Diagnostics and Caris Life Sciences). The rapidly developing genomic information is leading to the proliferation of MP services and assays and their subsequent introduction into clinical care.

One such MP service is the Caris Target Now ${ }^{\text {TM }}$. This service offers a new approach in which an evidence rated review of the literature based on the the US Preventive Task Force rating is utilized to identify targets in tumor tissue associated with current therapies [16]. Using a technology and platform agnostic approach, various targets are analyzed using a combination of assays such as gene sequencing, oligonucleotide microarray, mutational analyses, copy number changes using FISH analysis and protein expression by IHC.

This particular approach for MP to measure molecular targets was studied in a feasibility study in 2006 [17] and most recently in a multi-center clinical trial, across nine different cancer centers in the US. Using the Caris Life Sciences Caris Target Now ${ }^{\mathrm{TM}}$ MP service, Von Hoff et al. [18] reported a longer PFS for patients on MP-directed therapy than physician choice for $27 \%$ of patients $(95 \% \mathrm{CI}$,
17-38 \% $P=0.007)$. This study used a novel study design in which the patient served as their own controls and PFS ratio was determined by actual comparison of PFS on MP therapy versus PFS on patient's last prior therapy. For the participants $(18 / 66)$ who had a $P F S \geq 1.3$ overall survival was 9.7 months compared to 5 months on physician directed therapy. Interestingly, MP of tumors yielded actionable targets in $98 \%$ by this assay indicating that such an approach is feasible. However, it is to be noted that the targets identified may involve off-label use of therapies [18]. Whereas Von Hoff et al. study was restricted to advanced stage patients with metastases and refractory tumors, the approach may have significant benefits when used earlier.

Using the same Caris Target Now ${ }^{\text {TM }}$ service, ShachamShmuel et al. [19], reported two patients with advanced stage colon cancers in which identification of a target MGMT by IHC with this assay, led to measurable response to temozolomide treatment with decrease in serum markers and tumor shrinkage on CT. Using the same assay, discovery of targets was also reported in a interdigitating reticulum cell sarcoma, an exceedingly rare tumor [20]. Tsimberdou et al. [21] presented the MD Anderson experience using MP. Median time to treatment failure (TTF) in 161 patients with one aberration treated with matched targeted therapy was 5.3 months (95\% CI: 4.1, 6.6) versus 3.2 months (95\% CI: 2.9-4.0) for their prior systemic antitumor therapy (prior to referral to phase I) $(P=0.0003)$. For patients with one aberration, the CR-PR rate was $29 \%$ with matched targeted therapy versus $8 \%$ without matching $(P=0.0001)$. The $\mathrm{CR}+\mathrm{PR}$ rate was $6 \%$ in 438 patients without molecular testing treated on the same studies. Interestingly, these rates compare favorably with those reported by Von Hoff et al. for the Caris Target Now service. These preliminary results suggest that in early clinical trials, matching patients with targeted drugs based on their molecular profile results in (a) longer TTF compared to their prior therapy and (b) higher rates of response, survival and TTF compared to those seen in patients treated without molecular matching. The Battle trial for personalizing therapy for lung cancer identified targets of high interest in treatment of lung cancer and using an adaptive randomized trial utilized real time biomarker analyses to predict sensitivity or resistance to targeted agents [22]. A similar trial I-Spy 2 also employs this groundbreaking clinical trial model that uses genetic or biological markers ("biomarkers") from individual patient's tumors to screen promising new treatments, identifying which treatments are most effective in specific types of patients [23]. In addition, this innovative adaptive trial design similar to Battle Trial will enable researchers to use early data from one set of patients to guide decisions about which treatments might be more useful for patients later in the trial, and eliminate ineffective treatments more quickly. 
With more focus on the MP of tumors and greater realization of the limitations of one-biomarker-one target approach, cancer treatment in the US is about to experience a major revolution. Upfront MP of tumors at the time of diagnosis and subsequently at all points of tumor recurrence, whether local or distant, will change the treatment of oncology care forever. This will hopefully lead to better control of cancer, improved outcomes for patients, and a more rational and less expensive oncology care.

Open Access This article is distributed under the terms of the Creative Commons Attribution License which permits any use, distribution, and reproduction in any medium, provided the original author(s) and the source are credited.

\section{References}

1. Ross JS (2011) Cancer biomarkers, companion diagnostics and personalized oncology. Biomarker Med 5(3):277-279

2. Macconaill LE, Garraway LA (2010) Clinical implications of the cancer genome. J Clin Oncol 28(35):5219-5228

3. Schilsky RL, Allen J, Benner J, Sigal E, McClellan M (2010) Commentary: tackling the challenges of developing targeted therapies for cancer. Oncologist 15(5):484-487

4. Dhani N, Tu D, Sargent DJ et al (2009) Alternate endpoints for screening phase II studies. Clin Cancer Res 15:1873-1882

5. Smith S, Su D, Rigault de la Longrais IA et al (2007) ERCC1 genotype and phenotype in epithelial ovarian cancer identify patients likely to benefit from paclitaxel treatment in additional to platinum-based therapy. J Clin Oncol 25:5172-5179

6. Iwao-Koizumi K, Matoba R, Ueno N et al (2005) Prediction of docetaxel response in human breast cancer by gene expression profiling. J Clin Oncol 23:422-431

7. Cobo M, Isla D, Massuti B et al (2007) Customizing cisplatin based on quantitative excision repair cross complementing 1 mRNA expression: a phase III trial in non-small-cell lung cancer. J Clin Oncol 25:2747-2754

8. Kamel-Reid S, Zhang T, Persons DL, Nikiforova MN, Halling KC (2012) Validation of KRAS testing for anti-EGFR therapeutic decisions for patients with metastatic colorectal carcinoma. Arch Pathol Lab Med 136(1):26-32

9. Andre F, Mazouni C, Hortobagyi GN et al (2006) DNA arrays as predictors of efficacy of adjuvant/neoadjuvant chemotherapy in breast cancer patients: current data and issues on study design. Biochim Biophys Acta 1766:197-204
10. Sørlie T, Perou CM, Fan C et al (2006) Gene expression profiles do not consistently predict the clinical treatment response in locally advanced breast cancer. Mol Cancer Ther 5:2914-2918

11. Chang JC, Makris A, Gutierrez MC (2008) Gene expression patterns in formalin-fixed, paraffin embedded core biopsies predict docetaxel chemosensitivity in breast cancer patients. Breast Cancer Res Treat 108:233-240

12. Dressman HK, Berchuck A, Chan G et al (2007) An integrated genomic-based approach to individualized treatment of patients with advanced-stage ovarian cancer. J Clin Oncol 25:517-525

13. Kihara C, Tsunoda T, Tanaka T et al (2001) Prediction of sensitivity of esophageal tumors to adjuvant chemotherapy by cDNA microarray analysis of gene expression profiles. Cancer Res 61: 6474-6479

14. Del Rio M, Molina F, Bascoul-Mollevi C et al (2007) Gene expression signature in advanced colorectal cancer patients select drugs and response for the use of leucovorin, fluorouracil, and irinotecan. J Clin Oncol 25:773-780

15. Minna JD, Girard L, Xie Y (2007) Tumor mRNA expression profiles predict responses to chemotherapy. J Clin Oncol 25: 4329-4336

16. Harris T, Atkins D et al (2001) Current methods of the U.S. preventive service task force. Am J Prev Med 20:21-35

17. Von Hoff DD, Penny R, Shack S et al (2006) Frequency of potential therapeutic targets identified by immunohistochemistry (IHC) and DNA microarray (DMA) in tumors from patients who have progressed on multiple therapeutic agents. J Clin Oncol 24: 138 (suppl; abstr 3071)

18. Von Hoff DD, Stephenson JJ, Rosen PJ et al (2009) A pilot study utilizing molecular profiling of patients' tumors to find potential targets and select treatments for their refractory cancers. AACR Abstract, LB-259

19. Shacham-Shmueli E et al (2011) Response to temozolomide in patients with metastatic colorectal cancer with loss of MGMT expression: a new approach in the era of personalized medicine? J Clin Oncol 29(10):e262-e265

20. Weiss GJ, Alardon A, Halepota M et al (2010) Molecular characterization of interdigitating cell sarcoma. Rare Tumors. doi: $10.4081 /$ rt.2010.e50

21. Tsimberdou et al

22. Kim ES, Herbst RS, Lee JJ et al (2009) Phase II randomized study of biomarker-directed treatment for non-small cell lung cancer (NSCL): the BATTLE (Biomarker-Integrated Approaches of Targeted Therapy for Lung Cancer Elimination) clinical trial program. J Clin Oncol 27:412 (suppl; abstr 8024)

23. Barker AD, Sigman CC, Kelloff GJ, Hylton NM, Berry DA, Esserman LJ (2009) I-SPY 2: an adaptive breast cancer trial design in the setting of neoadjuvant chemotherapy. Clin Pharmacol Ther 86:97-100 